Cahiers $d u$ MONDE RUSSE

\section{Cahiers du monde russe}

Russie - Empire russe - Union soviétique et États indépendants

$53 / 4 \mid 2012$

Varia

\title{
Stephen J. Collier, Post-Soviet Social
}

\section{Frédéric Keck}

\section{OpenEdition \\ Journals}

Édition électronique

URL : http://journals.openedition.org/monderusse/7881

DOI : 10.4000/monderusse.7881

ISSN : $1777-5388$

Éditeur

Éditions de l'EHESS

Édition imprimée

Date de publication : 15 décembre 2012

ISSN : $1252-6576$

\section{Référence électronique}

Frédéric Keck, «Stephen J. Collier, Post-Soviet Social », Cahiers du monde russe [En ligne], 53/4 | 2012, mis en ligne le 08 octobre 2013, Consulté le 25 septembre 2020. URL : http://journals.openedition.org/ monderusse/7881 ; DOI : https://doi.org/10.4000/monderusse.7881

Ce document a été généré automatiquement le 25 septembre 2020

(c) École des hautes études en sciences sociales 


\title{
Stephen J. Collier, Post-Soviet Social
}

\author{
Frédéric Keck
}

\section{RÉFÉRENCE}

Stephen J. ColLIER, Post-Soviet Social. Neoliberalism, Social Modernity, Biopolitics.

Princeton : Princeton University Press, 2011, 320 p.

1 Ce livre ne porte pas tant sur la Russie que sur le néo-libéralisme comme «style de raisonnement ». Stephen Collier est anthropologue à la New School for Social Research, élève de Paul Rabinow à l'université de Berkeley, et il se réclame explicitement d'une approche foucaldienne. Il considère la Russie postsoviétique comme un "cas » pour une réflexion plus générale sur les formes de la gouvernementalité contemporaine. L'étude s'enracine dans l'ethnographie d'une ville moyenne industrielle, Belaja Kalitva, dans la province de Rostov. Cependant elle déborde en permanence ce cadre par la consultation de plans de construction de l'époque soviétique et de plans de réforme de l'ère postsoviétique.

2 L'enquête de Stephen Collier part du constat suivant: les villes moyennes industrielles constituant la majeure partie du tissu urbain russe (60\% de la population urbaine vivait dans des villes de moins de 500000 habitants en 1990 et $30 \%$ dans des villes de moins de 100000 habitants), ont été au cœur des projets modernisateurs de l'époque soviétique et postsoviétique. L'étude des villes moyennes révèle ainsi selon lui des continuités entre ces deux périodes. Selon Collier, la menace d'effondrement liée à la période postsoviétique a mis en lumière en les fragilisant des infrastructures construites lors de la période soviétique. L'enquête de Collier porte sur ces infrastructures, non tant dans l'optique marxiste d'un renversement des superstructures idéologiques vers les infrastructures matérielles, mais plutôt, dans une optique foucaldienne, pour voir comment celles-ci sont problématisées par des experts en gouvernementalité. "The more time I spent in Russia, the more I became convinced that budgets, spending norms, pipes, and wires were the sites where the relationship between Soviet social modernity and neoliberal reforms could be examined. » (p. 8) 
3 La méthode de Stephen Collier est donc proche de la sociologie des sciences de Michel Callon et Bruno Latour. Là où des auteurs critiques (David Harvey ou Naomi Klein) reprochent au néo-libéralisme de détruire de façon catastrophique des formes du social supposées déjà là, Stephen Collier montre comment ces formes du social émergent d'une composition contingente entre des actions locales, ce qu'il a appelé avec Aiwah Ong des «assemblages $»^{1}$. Mais à la différence de la sociologie des sciences, l'anthropologie de la modernité ne suppose pas que la question du mode d'existence du social est soulevée de l'extérieur par le sociologue: elle examine comment cette question est posée par les acteurs lorsqu'ils font des projets. Collier se demande comment la modernité sociale est devenue un problème pour des experts russes chargés des réformes à l'ère soviétique et postsoviétique. D'où l'intérêt pour les infrastructures comme dimension à la fois fragile et résistante du tissu urbain, et la focalisation « micropolitique » sur les tuyaux et les canalisations comme révélateurs du social.

4 Le livre se divise en deux parties. Dans la première, Collier examine les politiques urbaines en Russie à la lumière du schéma foucaldien du passage du «pouvoir souverain» à la "biopolitique». Si Foucault étudiait cette transformation dans le discours économique des physiocrates à la fin du dix-huitième siècle, Collier la repère dans le contraste entre les projets urbains de Pierre Le Grand et ceux qui sont formulés dans les années 1920-1930. Au projet pétrovien de contrôle administratif de la ville sur le modèle militaire, poursuivi selon lui jusqu'à la fin du régime tsariste, succède le projet soviétique de mise en valeur de la production dans toutes ses composantes, dont la nouveauté se marque dans la planification nationale. Le terme "hozjaistvo» fait l'objet de nombreux commentaires pour montrer comment son double sens de production et d'accomplissement des besoins s'étend à tous les domaines de la vie sociale. Collier refuse donc le schéma du contrôle totalitaire pour voir comment ces projets portant sur les infrastructures de la production créent un "paysage moral ", c'est-à-dire un espace dans lequel des relations de soin deviennent possibles. La biopolitique n'est pas à ses yeux l'envers du régime totalitaire mais un espace de gouvernementalité qui considère comme centrales les questions de santé et de bienêtre (welfare).

5 La seconde partie est consacrée aux projets de réforme néo-libérale dans la période postsoviétique. Alors que les experts russes étaient au cœur de la première partie, ce sont plutôt ceux de la Banque mondiale qui font l'objet de la seconde partie. Les théories de James Buchanan et George Stigler sur la politique budgétaire et fiscale sont tout particulièrement examinées. Elles considèrent en effet l'État et le marché comme deux façons de produire la santé et le bien-être qui doivent être comparées dans leur efficacité. Alors que les critiques du néo-libéralisme le présentent comme un remplacement de l'État bienveillant par les forces obscures du marché, Collier affirme que les théories de Buchanan et Stigler posent de façon nouvelle le problème de la justice dans un contexte biopolitique : comment redistribuer les biens fondamentaux en tenant compte de l'accès inégal des individus à ces biens selon les lieux où ils résident? "They invented a new empiricity, a new principle of reality, which made it possible to grasp the minute details of bureaucratic arrangements or material infrastructures in a new and productive way. » (p. 246)

6 Collier montre ensuite comment cette nouvelle problématique oriente le programme postsoviétique de distribution du chauffage, qui pose pour la première fois la question 
d'une demande différenciée de chauffage dans un pays où l'offre est abondante. La reconversion des tuyaux soviétiques dans le nouveau système de chauffage permet donc de nuancer le portrait d'une Russie postsoviétique qui s'est effondrée sous les coups du néo-libéralisme. "Reformed or not, the heat apparatus will serve as a great schock absorber during economic downturn, weaving together the elements of the citybuilding khoziaistvo as a sticky nexus of pipes, hot water and warm bodies, in what remains, as such, a very old country. » (p. 244)

7 Ce livre propose donc une élaboration particulièrement féconde de la notion d'infrastructure, qui a souvent été écartée des sciences politiques, notamment dans une approche foucaldienne de la gouvernementalité. Elle a permis à Stephen Collier d'apporter ensuite un éclairage nouveau sur les formes contemporaines de la "biosécurité », comprises comme des réorganisations des infrastructures vitales dans la perspective d'une catastrophe possible ${ }^{2}$. L'analyse de la biopolitique est ainsi orientée vers les rationalisations nouvelles davantage que vers l'expérience qui en est faite et les résistances qu'elles suscitent. On peut s'étonner ainsi de voir que l'analyse des projets des experts évacue l'expérience des habitants des villes, et plus encore lorsque des experts non-russes transforment les conditions de l'habitat russe. Mais on peut aussi penser qu'en considérant les villes moyennes comme des lieux de réflexivité, sans passer par le niveau de l'État central, Stephen Collier propose une nouvelle articulation entre anthropologie et histoire du monde russe.

\section{NOTES}

1. Cf. S.J.Collier et A. Ong, Global Assemblages: Technology, Politics, and Ethics as Anthropological Problems, Malden, MA : Blackwell, 2005.

2. S.J. Collier, A. Lakoff et P. Rabinow, "Biosecurity: Towards and anthropology of the contemporary", Anthropology Today, 20 (5), 2004, p.3-7; S.J. Collier et A. Lakoff, "The Vulnerability of Vital Systems: How "Critical Infrastructure" Became a Security Problem" in M. Dunn et K.S. Kristensen, eds., The Politics of Securing the Homeland: Critical Infrastructure, Risk and Securitisation Londres : Routledge, 2008 ; S. Collier et A. Lakoff, dir., Biosecurity Interventions: Global Health and Security in Question, New York : SSRC-University of Columbia Press, 2008. 